\title{
Differences in Host Immune Populations Between Rhesus Macaques and Cynomolgus Macaque Subspecies, in Relation to Susceptibility to Mycobacterium Tuberculosis Infection.
}

\author{
Laura Sibley ( $\sim$ Laura.sibley@phe.gov.uk) \\ Public Health England \\ Owen Daykin-Pont \\ Public Health England \\ Charlotte Sarfas \\ Public Health England \\ Jordan Pascoe \\ Public Health England \\ Alexandra Morrison \\ Public Health England \\ Andrew D. White \\ Public Health England \\ Sally Sharpe \\ Public Health England
}

\section{Research Article}

Keywords: Macaque, rhesus, cynomolgus, immunology, lymphocytes, monocytes, blood, tuberculosis

Posted Date: March 1st, 2021

DOI: https://doi.org/10.21203/rs.3.rs-252046/v1

License: (c) (i) This work is licensed under a Creative Commons Attribution 4.0 International License. Read Full License

Version of Record: A version of this preprint was published at Scientific Reports on April 23rd, 2021. See the published version at https://doi.org/10.1038/s41598-021-87872-x. 


\begin{abstract}
Rhesus (Macaca mulatta) and cynomolgus (Macaca fasicularis) macaques of distinct genetic origin are understood to vary in susceptibility to Mycobacterium tuberculosis, and therefore differences in their immune systems may account for the differences in disease control. Monocyte:lymphocyte $(\mathrm{M}: \mathrm{L})$ ratio has been identified as a risk factor for M. tuberculosis infection and is known to vary between macaque species. We aimed to characterise the constituent monocyte and lymphocyte populations between macaque species, and profile other major immune cell subsets including: $\mathrm{CD}^{+}{ }^{+}$and $\mathrm{CD} 8^{+} \mathrm{T}$-cells, NK-cells, B-cells, monocyte subsets and myeloid dendritic cells. We found immune cell subsets to vary significantly between macaque species. Frequencies of $\mathrm{CD} 4^{+}$and $\mathrm{CD} 8^{+}$T-cells and the CD4:CD8 ratio showed significant separation between species, while myeloid dendritic cells best associated macaque populations by $M$. tuberculosis susceptibility. A more comprehensive understanding of the immune parameters between macaque species may contribute to the identification of new biomarkers and correlates of protection.
\end{abstract}

\title{
Introduction
}

Non-human primates are widely used in infectious disease research because of the similarity in immune system and physiology to humans. Several genetically distinct populations within different macaque species are available for research use, and which population/species is chosen may depend on several factors; for example, their suitability for certain infectious diseases, and ease of availability.

At Public Health England (PHE), four types of macaque have been used in Tuberculosis (TB) research; rhesus macaques (Macacca mulatta) of Indian genotype and cynomolgus macaques (Macacca fasicularis) of Mauritian (MCM) or Asian (Indonesian (ICM) and Chinese (CCM)) genotype. Macaques show a spectrum of TB disease, similar to humans ${ }^{1,2}$, but it is well known that the populations have differences in susceptibilities to TB disease when infected with Mycobacterium tuberculosis (M. tb); MCM and RM are more susceptible to TB than $\mathrm{CCM}^{3}$ and ICM ${ }^{4}$.

The reasons behind differences in the populations are unclear, although correlates of TB risk identified in human populations may also be applicable to macaque species. For example, our previous work had shown that monocyte:lymphocyte ratio (M:L) did differ between populations. A high, or extremely low $\mathrm{M}: \mathrm{L}$ has been shown in humans to associated with risk to development of $\mathrm{TB}^{5}$, and in our research we have shown that RM and MCM have significantly higher $\mathrm{M}: \mathrm{L}$ ratios than $\mathrm{CCM}^{6}$.

To further investigate differences in the host immune systems of macaque populations, a flow cytometric immunophenotyping assay was developed to compare T-cell, NK cell and monocyte subsets between populations as reports suggest that monocyte subsets and NK cells have the potential to bias the immune system to influence susceptibility to $\mathrm{TB}^{5,7}$.

The aim of this study was to characterise and compare these different host factors across rhesus and cynomolgus macaque species and between cynomolgus macaques of distinct genetic origin, and to identify differences between them, and determine whether these may have a role in defining their susceptibility to TB through a retrospective analysis of data from TB infection studies.

\section{Results}

\section{Immune cell differences between genetically distinct macaque populations as measured with the haematology analyser}

Extensive differences between immune cell populations were observed between the species (Fig. 1). Rhesus macaques possessed significantly lower numbers of lymphocytes than all cynomolgus macaque populations (ICM $(p<0.001)$, CCM $(p<0.001)$ and MCM ( $p=0.001)$ (Fig. 1A). The number of lymphocytes counted in the ICM was significantly higher than in the $\operatorname{MCM}(p=0.034)$ (Fig. 1A).

The RM population also had the fewest monocytes (Fig. 1B), with levels significantly less than those measured in ICM $(p<0.001)$, and MCM $(p=0.004)$. Furthermore, the ICM population showed a significantly higher monocyte count than both other cynomolgus populations (MCM $p=0.011, \mathrm{CCM} p=0.001)$.

Indonesian Cynomolgus macaques had significantly fewer neutrophils than all other populations (CCM $(p<0.001)$. RM $(p=0.002)$ and MCM $(p=0.004)$ ) (Fig. 1C). The ICM population also displayed the least variation in neutrophil count; with both RM, MCM and CCM displaying considerable spread within each population. The CCM population displayed significantly higher neutrophil counts compared to RM $(p=0.025)$.

RM and CCM possessed the fewest eosinophils (Fig. 1D). The ICM and MCM populations displayed a higher count and greater spread of data within their populations. Rhesus macaques had significantly fewer eosinophils than both MCM and ICM (both $p<0.001$ ). The CCM population had significantly fewer eosinophils than ICM $(p=0.002)$, but not significantly lower than RM or MCM (Figure, 1D).

Chinese Cynomolgus macaques had a signifcantly lower M:L than RM $(\mathrm{p}=0.0001)$, MCM $(p=0.0010)$, and ICM $(p=0.001)$ (Fig. 1E). However, none of the other differences in M:L reached significance. The spread in values was greatest in RM, although this may be due to the larger sample size assessed ( $n=84)$. Indonesian Cynomolgus macaques showed significantly lower N:L compared with RM $(p<0.001)$, MCM $(p=0.020)$ and CCM ( $p=0.043)($ Fig. $1 \mathrm{~F})$. No other significant differences were observed in N:L between macaque sub-species.

\section{Cell frequencies as measured using immunophenotyping of PBMCs}


To look in more detail at cell populations and subsets, cryopreserved PBMCs from all macaque populations were used to characterise the key immune cell populations.

We observed the ICM population to have a significantly higher frequency of $\mathrm{CD} 3^{+}$T-lymphocytes as a proportion of total single events, when compared to RM $(p=0.013)$ (Fig. 2A) (gating strategy in supplementary Fig. 2). The proportion of CD $4^{+}$and $C D 8^{+}$subsets within the CD $3+$ population varied according to macaque species and genetic origin, with MCM having the highest proportion of CD8 ${ }^{+}$and RM having the highest $\mathrm{CD} 4^{+}$population (Fig. $\left.2 \mathrm{~B}\right)$. The $\mathrm{CD} 4: \mathrm{CD} 8$ ratio was also significantly raised in rhesus compared with $\mathrm{MCM}(p<0.001)$, ICM $(p=0.001)$ and CCM $(p=0.001)$ (Supplementary Data 1). Indeed, the frequency of $\mathrm{CD} 4^{+}$T-lymphocytes was significantly higher in RM compared to MCM $(p<0.001)$, ICM $(p=0.001)$ and CCM ( $\left.p=0.016\right)($ Supplementary data). Relatively low frequencies of double positive $\left(C D 4^{+} C D 8^{+}\right)(D P)$ T-lymphocyte populations were detected in all macaque groups; although, these were most apparent in MCM and CCM populations where frequencies of DP T-cells were greater than in rhesus ( $\mathrm{p} \leq 0.001$ for both MCM and CCM) or the ICM group, although the later comparisons did not reach significance (Supplementary data 1).

We found the total proportions of monocytes not to vary between species (Fig. 2C) (gating strategy in supplementary Fig. 3). However, we did observe significant differences in the proportions of different monocyte phenotypes. Rhesus macaques possessed the highest frequency of $\mathrm{CD}_{14}^{+}{ }^{+}$monocytes overall (Fig. 2D). The MCM population had a considerably lower proportion of CD14 $4^{+}$monocytes compared to the rhesus $(p=0.004)$ and CCM $(p=0.014)$ populations. Significant differences were not seen in frequencies of the $\mathrm{CD} 14^{+} \mathrm{CD} 16^{+}$(Intermediate) population. The ICM and CCM populations displayed the highest frequencies of the $\mathrm{CD}^{-} 4^{-} \mathrm{CD} 16^{+}$monocyte (Fig. 2D), which were significantly higher compared to $\mathrm{RM}(\mathrm{ICM}(p=0.039)$ and CCM $(p=0.004))$ (Supplementary data).

Differences in NK cell frequency (CD159a+) were apparent between rhesus and cynomolgus species (Fig. 2E) with fewer NK cells measured in rhesus macaques relative to all cynomolgus subspecies. This result was significant when compared to the MCM $(p=0.032)$ and ICM $(p=0.010)$ populations (Fig. 2E) (gating strategy in supplementary Fig. 4). Natural Killer (NK) cell populations are typically subdivided into cytotoxic and immunomodulatory phenotypes based on expression of the surface markers CD16 (cytotoxic) and CD56 (immunomodulatory) ${ }^{8}$. The phenotypic profile of NK subsets measured in MCM and CCM were similar as both had relatively high proportions of CD56 ${ }^{+}$and DP populations in comparison to rhesus, and ICM in which the fewest CD56 expressing NK-cells were detected. Cytotoxic $\left(C D 16^{+} \mathrm{CD}_{5}{ }^{-}\right)$NK-cells were most abundant in rhesus macaques with proportions significantly higher than in MCM $(p=0.0421)$ and CCM $(p=0.002)$. Similarly, cytotoxic NK-cells were more prevalent in ICM than in CCM $(p=0.006)$ (Supplementary data 1$)$.

NK T-cells, an unconventional CD3 ${ }^{+} \mathrm{CD}_{16} 6^{+}$lymphocyte and antigen presenting CD11 $\mathrm{c}^{+}$B-cells, were not found to significantly differ between macaque species (Fig. $2 \mathrm{G}$ and H). Monocyte derived Dendritic Cells ( $\mathrm{mDC}$ ) were significantly higher in both ICM $(p=0.034)$ and CCM ( $p=0.026)$ when compared to rhesus (Fig. 2l).

\section{Relationships between cell populations and associations with TB disease}

Principle Component Analysis (PCA) was applied to immunophenotyping datasets as a multivariate analysis technique for the identification of cellular immune compartment variables that differentiated between macaque species and sub-species (Fig. 3).

Along dimension 1, the factors that account for $25.4 \%$ (eigenvalue of 3.3 ) of the variance in the data were classical and non-classical monocytes, pathologybased scores and $\mathrm{CD} 4^{+} \mathrm{T}$-cells, with $\mathrm{CD} 4^{+}$T-cells clustering with pathology scores. Dimension 2 , which accounted for $21.1 \%$ of the variance (eigenvalue of 2.75) were affected mostly by $\mathrm{CD} 8^{+}$T-cells, $\mathrm{CD} 16^{+}$Classical monocytes and CD $4^{+}$T-cells (Fig. $3 \mathrm{~A}$ and supplementary data 5 and 6 ).

Looking at the distribution of different populations of macaques, we can see that each population of macaques cluster separately, with RM having the largest variation and overlapping with MCM and CCM. ICM and RM show little overlap, and there was also little overlap between MCM and CCM. Nonclassical monocytes and $\mathrm{CD} 8^{+}$T-cells seem to distinguish ICM from RM, and CD8 ${ }^{+}$and classical monocytes from CCM and MCM (Fig. 3B).

As T-cells appeared to be the most discriminatory between populations, we analysed the relationship between these cells and pathology score, calculated as described by Sharpe et al $^{9}$. This was used to investigate the potential for a common relationship between T-cells prior to infection and pathology score after infection with $M$. $t b$. We found that the CD $4^{+}$T-cells at baseline did correlate with an increased pathology score $(r=0.527, p=0.025)($ Fig. 3C). There was a non-significant trend for $\mathrm{CD}^{+}$T-cells to negatively correlate with pathology score $(r=0.445, p=0.064)$ (Fig. 3D). Classical and non-classical monocytes were not found to correlate with pathology score $(r=0.270, p=0.278$ and $r=-0.111, p=0.661$ respectively).

\section{Discussion}

In this study, two approaches were taken to characterise, enumerate and compare the levels of white cell populations in the blood of four genetically distinct macaque populations. Although there are limited reports comparing the immune cells of humans with those in certain macaque populations ${ }^{10,11}$ to our knowledge, this is the first time a direct comparison of four genetically distinct macaque populations has been performed using the same assays.

Evaluation of the cellular composition of anti-coagulated blood using a haematology analyser enables an unbiased analysis of cell population frequency and number per $\mathrm{ml}$ of blood for all types of cells present. Conversely, data originating from PBMC samples relates to mononuclear cell populations only and is proportional, but the main advantages of flow cytometric immunophenotyping is that it can generate more detailed data and can be applied to archived samples enabling retrospective interrogation of materials. 
The haematology analyser derived data set demonstrated that lymphocytes and monocyte counts were different between rhesus and cynomolgus, whereas there was little difference between the genetically distinct cynomolgus populations. Separating the populations into TB disease susceptible (RM and MCM) and less susceptible (ICM and CCM), it was only lymphocytes levels that were different between these groupings. Eosinophil and neutrophils counts were different between groups, but the differences were not common between the susceptible and less susceptible groups and so, it is perhaps unlikely that basal eosinophils and neutrophil numbers have a significant bearing on susceptibility to TB disease.

Immunophenotyping studies of PBMC were used to evaluate the subtypes of the lymphocytes and monocytes present. When the least susceptible populations (ICM and CCM) were grouped and compared to the susceptible populations (RM and MCM), key differences were identified in the CD4:CD8 ratio and $\mathrm{mDC}$ proportions.

CD4 ${ }^{+}$T-cells were identified using PCA to discriminate between the macaque populations and CD $4^{+}$T-cells also correlated with TB-induced disease burden measured using a pathology-based scoring system. Although it is known that CD $4^{+}$T-cells are necessary for control of TB infection, as CD $4^{+}$cell depletion due to HIV is a risk factor for TB infection ${ }^{12}$; HIV is a disease state with an abnormally low number of CD $4^{+}$T-cells whereas these are healthy animals so in normal conditions it appears that high numbers of $C D 4^{+}$T-cells may impact on TB disease progression. There are also many subtypes of $C D 4^{+} \mathrm{T}$-cells to be considered and this analysis may be too simplistic, and subtypes and activation status also need to be taken into account in future analysis.

$\mathrm{CD}^{+}$T-cells and $\mathrm{CD} 16^{+} \mathrm{NK}$ cells contributed to the PCA second dimension, highlighting a potential difference in cells that have cytolytic roles between primate populations. We saw a non-significant correlation between higher numbers of $\mathrm{CD}^{+}$cells before infection that correlated with lower pathology scores. In $\mathrm{CD}^{+}$depletion studies in primates, $\mathrm{CD} 8^{+}$T-cells have been shown to be important in the control of infection ${ }^{13}$. The CD4:CD8 ratios defined in ICM and CCM showed a balance in $\mathrm{CD} 4^{+}$and $\mathrm{CD} 8^{+}$T-cells, whereas in MCM a skew towards the CD8 population was found, in line with the previous report from Zitsman et $\mathrm{al}^{14}$, whereas the populations in RM were more biased towards CD4. A low CD4:CD8 ratio has been found to be a predictor TB in HIV patients ${ }^{15}$ so as MCM do have the lowest CD4:CD8 ratio, this could be a contributing risk factor in their susceptibility to TB.

NK cell transcripts were found to be lower in CMV + infants, that went on to develop TB ${ }^{7}$ suggesting a link between NK cells and TB susceptibility. A study comparing NK subsets between persons from a TB endemic country with TB naïve persons showed that there was little difference in the frequency of cytolytic NK cells, but that those NK cells had different reactivity and functional capacity ${ }^{16}$. Therefore, in this study, the NK subtype proportions were most similar between MCM and CCM which have very different susceptibilities to TB so further investigation into the functionality of the NK cells is required to determine whether there are differences in their capacity to react to TB and influence disease progression.

Monocytes contributed to the variance in the first dimension in the PCA, and there was a difference in the proportion of CD14 ${ }^{+}$classical monocytes between rhesus and cynomolgus macaques, but they did not correlate with pathology. Dijkman et al saw differences in monocyte subtypes and cytokine production post-infection with TB between rhesus and cynomolgus macaques ${ }^{17}$ and so looking post-infection at differences whether there are differences between populations in how they respond to infection that relates to their basal subtypes would be something to examine further in future work.

Antigen presenting cells (APCs) such as mDCs are a key component of T-cell activation, and this population was present at significantly higher frequencies in the macaque populations that are less susceptible to TB. Efficient priming of T-cells is considered to be key in protecting against TB and TB modulates DC activity by delaying their ability to migrate to the lymph nodes, hampering the formation of an effective immune response and giving the TB infection time to establish ${ }^{18}$. Furthermore DCs have been found to be present at lower levels in patients with TB ${ }^{19}$. Having a higher number of mDCs has the potential to confer an advantage by increasing the likelihood of migration to the lymph nodes and increasing the interactions with T-cells to promote an early immune response to infection. Neutrophils have an important role to shuttle bacteria to mDCs and enhance antigen presentation, and TB has been shown to affect neutrophil apoptosis, leading to less efficient DC priming ${ }^{20}$ and so the higher numbers of neutrophils in the CCM, coupled with higher numbers of mDCs may be contributing factors to their control of TB.

Overall, these studies have revealed differences in the cellular composition of peripheral blood in four genetically distinct macaque populations, and particularly between rhesus and cynomolgus macaques in terms of lymphocyte populations. The concordance of findings from haematology analyser-based and flow cytometry-based measurements, supports the concept that there are fundamental differences in the makeup of the immune systems of these species. Others have noted that macaques vary genetically substantially between geographical locations ${ }^{21}$, and recommend caution when comparing data from different models for the same diseases as contributing factors could obscure risk factor-disease associations, or lead to artificial associations. Therefore, it is important to understand the genetic background of the animals used in studies, together with the potential implications that any consequent constitutive differences between populations may have on the experimental outcome. Characterisation of macaque populations provides the opportunity to select populations with desirable characteristics for specific studies so differences can be exploited to further understand the factors required to promote a successful immune system.

\section{Methods}

\section{Use of animal tissue and ethical statement.}

The data presented was generated from samples collected from animals housed within the PHE-managed macaque breeding units and from macaques enrolled in previous studies at PHE Porton. 
The rhesus macaques (RM) and cynomolgus macaques were obtained from established UK Home Office approved breeding colonies in the United Kingdom (ICM, MCM, RM) and China (CCM). Genetic analysis of macaques from the UK colonies has previously confirmed the rhesus macaques to be of the Indian genotype and cynomolgus macaques of Mauritian ${ }^{22}$ or Indonesian ${ }^{23}$ genotypes. The numbers of each species used for each type of analysis is outlined in Table 1.

Animals were housed in compatible social groups. In studies not involving challenge with $M$. tuberculosis, macaques were housed in pens designed to allow access to indoor and outdoor environments and measured approximately $20 \mathrm{~m}^{2}$ by $2.5 \mathrm{~m}$ high for indoor-, and $37.8 \mathrm{~m}^{2}$ by $3.7 \mathrm{~m}$ high for outdoor-enclosures. Pens were constructed with a range of high-level observation balconies and a floor of deep litter was provided in internal enclosures to allow foraging. Following challenge with $M$. tuberculosis macaques were housed in cages constructed with high level observation balconies with the housing environment was maintained within a temperature range of $18-24{ }^{\circ} \mathrm{C}$ with a relative humidity range of 40 to $70 \%$. Additional environmental enrichment was afforded by the provision of toys, swings, climbing apparatus, feeding puzzles and DVDs for visual stimulation. In addition to standard old-world primate pellets, diet was further supplemented with a selection of fresh vegetables and fruit. For each procedure, sedation was applied by intramuscular injection with ketamine hydrochloride (10 mg/kg) (Ketaset, Fort Dodge Animal Health Ltd, Southampton, UK). All procedures were in line with the Home Office (UK) Code of Practice for Housing and Care of Animals Used in Scientific Procedures (1989), and the National Committee for Refinement, Reduction and Replacement (NC3Rs) Guidelines on Primate Accommodation, Care and Use, August 2006. All animal procedure and study design were approved by the Public Health England, Animal Welfare and Ethical Review Body, Porton Down UK, and authorised under an appropriate UK Home Office project licence and all of the authors complied with ARRIVE guidelines.

Table 1

Number of samples from each population for each type

\begin{tabular}{|lllll|}
\hline & RM & MCM & ICM & CCM \\
\hline Haematology analyser & 84 & 26 & 27 & 18 \\
\hline Flow cytometry & 20 & 19 & 8 & 15 \\
\hline Flow cytometry M:L & 10 & 6 & 6 & 6 \\
\hline TB infection studies & 6 & 2 & 4 & 6 \\
\hline
\end{tabular}

\section{IDEXX ProCyte DX}

Blood samples anti-coagulated with EDTA $(1.8 \mathrm{mg} / \mathrm{ml}$ blood) or heparin, (both BD Biosciences, USA). All ProCyte DX were analysed using the IDEXX ProCyte DX Haematology analyser (IDEXX, USA). Results are expressed as absolute counts $(\mathrm{K} / \mu \mathrm{l})$, or ratios thereof.

\section{PBMC Isolation and resuscitation}

Peripheral blood mononuclear cell (PBMC) samples were isolated using standard methods ${ }^{24}$. The density gradient material used for PBMC isolation was dependent on the macaque species; Ficoll Histopaque (GE Healthcare, USA) for rhesus macaques or Percoll (Sigma-Aldrich, UK) for cynomolgus macaques. Samples were stored at $-180^{\circ} \mathrm{C}$ in isothermal tanks prior to analysis.

Upon resuscitation for analysis, samples were washed twice with RPMI media (Sigma-Aldrich, UK) supplemented with 10\% foetal calf serum (FCS), with added DNase ( $1 \mathrm{Unit} \mathrm{ml}^{-1}$ ) (Sigma-Aldrich, UK) by centrifugation at $400 \mathrm{~g}$ for $5 \mathrm{mins}$. The samples were rested for either $2 \mathrm{hr}$, or overnight, at $37^{\circ} \mathrm{C}, 5 \% \mathrm{CO}_{2}$.

\section{Flow cytometric staining}

Following resting of cells, adherent cells were washed from the tube by addition of 2 mM EDTA (Sigma-Aldrich, UK) and gently agitated for 15 minutes. A viable cell count was performed, and $1.5 \times 10^{6}$ cells per animal were used for flow cytometric staining. LIVE/DEAD Fixable Dead Stain Kit Violet (Invitrogen, UK) was used according to manufacturer protocol, prior to application of other antibodies to reduce background staining; and was incubated for 30-minutes. Cells were then washed by centrifugation at $400 \mathrm{~g}$ for 5 mins and resuspended in PBS. Staining with the full panel of antibodies was applied and incubated for 30 minutes according to the information in Table 2. Lymphocytes and monocytes were initially gated using forward scatter (FSC) and side scatter (SSC). Lymphocytes were then determined using Live/Dead and $\mathrm{CD}_{20}^{-} \mathrm{CD}^{+}$staining and $\mathrm{CD} 4^{+}$and $\mathrm{CD} 8^{+}$staining from the $\mathrm{CD} 20^{-} \mathrm{CD} 3^{+}$population

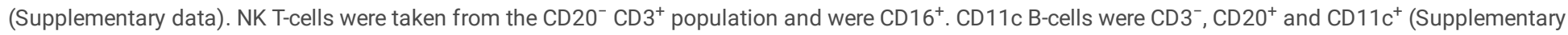
data). NK Cells were $C D 3^{-}, C D 8^{+} \mathrm{HLA}^{-D R^{-}}$and then $\mathrm{CD} 159 \mathrm{a}^{+}$. The subsets of NK cells were taken from the $\mathrm{CD} 159 \mathrm{a}^{+}$population and were defined as either $\mathrm{CD}_{16}{ }^{+} \mathrm{CD}^{-} 6^{-}$(cytotoxic), $\mathrm{CD} 16^{+} \mathrm{CD}^{+} 6^{+}$(intermediate) or $\mathrm{CD}^{-} 6^{-} \mathrm{CD}^{+} 6^{+}$(immunomodulatory) (Supplementary data). From the initial monocyte gate, monocytes were characterised by being $\mathrm{CD}^{-}$and $\mathrm{CD} 20^{-}$, then $\mathrm{CD} 14^{+/-}$and $\mathrm{HLA}^{-\mathrm{DR}}{ }^{+}$and subsets were defined using $\mathrm{CD} 14^{+} \mathrm{CD} 16^{-}(\mathrm{Classical}), \mathrm{CD}_{1} 4^{+} \mathrm{CD} 16^{+}$ (intermediate) and $\mathrm{CD}_{14}^{-} \mathrm{CD}^{+} 6^{+}$(non-classical) (Supplementary data). The mDCs were defined as CD14 ${ }^{-} \mathrm{CD}^{-}$, and then $\mathrm{CD}^{-} \mathrm{CD}^{-} 0^{-}$, followed by $^{-} \mathrm{CD} 159 \mathrm{a}^{-}$ gating, $\mathrm{HLA}_{-}-\mathrm{R}^{+}$gating and then characterised as being $\mathrm{CD} 16^{+}$and $\mathrm{CD} 11 \mathrm{c}^{+}$(Supplementary data). Following antibody labelling cells were washed twice in PBS by centrifugation at $400 \mathrm{~g}$ for 5 mins, resuspended in $4 \%$ paraformaldehyde solution and rested for a minimum of 20 minutes, prior to flow cytometric acquisition using LSRII Fortessa flow cytometer (BD Biosciences, Oxford, UK). 
Table 2

Antibody panel

\begin{tabular}{|c|c|c|c|c|c|c|c|c|c|c|c|}
\hline Antigens & CD3 & CD4 & CD8 & CD11c & CD14 & CD16 & CD20 & CD56 & CD159a & HLA-DR & Live/Dead \\
\hline Lymphocyte & CD3 & CD4 & CD8 & - & - & - & - & - & - & - & $\mathrm{L} / \mathrm{D}$ \\
\hline NK-cell & - & - & CD8 & - & - & CD16 & - & CD56 & CD159a & Dim/- & L/D \\
\hline Monocyte & - & - & - & - & CD14 & CD16 & - & - & - & HLA-DR & $\mathrm{L} / \mathrm{D}$ \\
\hline $\mathrm{mDC}$ & - & - & - & CD11c & - & CD16 & - & - & - & HLA-DR & $\mathrm{L} / \mathrm{D}$ \\
\hline B-cell & - & - & - & CD11c & - & - & CD20 & - & - & - & $L / D$ \\
\hline NKT Cell & CD3 & - & $\mathrm{CD} 8$ & - & - & CD16 & - & CD56 & - & - & $L / D$ \\
\hline Fluorochromes & AF700 & $\begin{array}{l}\text { PerCP- } \\
\text { Cy5.5 }\end{array}$ & $\begin{array}{l}\text { APC- } \\
\text { Fire750 }\end{array}$ & $\mathrm{PE}$ & APC & BV786 & $\begin{array}{l}\text { PE- } \\
\text { Dazzle }\end{array}$ & BV605 & PE-Cy7 & BUV395 & Violet \\
\hline Clone & $\mathrm{FN}-18$ & OKT4 & SK1 & 3.9 & M5E2 & $3 G 8$ & $2 \mathrm{H} 7$ & MY31 & Z199 & G46-6 & N/A \\
\hline Manufacturer & BD & Biolegend & Biolegend & Biolegend & Biolegend & Biolegend & Biolegend & $\mathrm{BD}$ & $\begin{array}{l}\text { Beckman } \\
\text { Coulter }\end{array}$ & BD & Invitrogen \\
\hline
\end{tabular}

\section{Data analysis}

IDEXX data was analysed using GraphPad Prism V8.0.1 (GraphPad Software Inc, USA) and the Kruskal-Wallis test for multiple comparisons was applied to data sets. Flow cytometry data was analysed using FlowJo V10 (BD Biosciences, UK) and data exported for analysis using GraphPad Prism V8.01. Data was tested for normality using Shapiro-Wilk test for normality and the Kruskall-Wallis tests with Dunn's corrections for multiple comparisons were applied to data. Principle Component Analysis (PCA) was carried out using RStudio version 3.5.3 (RStudio Inc., Boston, MA, USA).

\section{Declarations}

\section{Author contributions}

LS, ADW and CS contributed to the conceptualisation and methodology; ODP and AM performed experiments; ODP, LS and JP performed analysis; LS, ADW, CS and SS provided supervision; LS wrote the paper and all authors provided assistance and critical review. LS and ODP contributed equally to the work.

\section{Acknowledgements}

We thank the staff of the Biological Investigations Group at PHE Porton for assistance in conducting studies and we would like to thank Adam Mabbutt and Konstantinos Gkolfinos for technical expertise.

\section{Data availability statement}

All data generated or analysed during this study are included in this published article (and its Supplementary Information files).

\section{Ethics declaration}

All animal procedures and study design were approved by the Public Health England, Animal Welfare and Ethical Review Body, Porton Down, UK, and authorised under an appropriate UK Home Office project license.

\section{Competing interests}

All authors declare no competing interests.

\section{References}

1. Flynn, J. L. et al. Non-human primates: a model for tuberculosis research. Tuberculosis (Edinb). 83, 116-8 (2003).

2. Capuano, S. V et al. Experimental Mycobacterium tuberculosis infection of cynomolgus macaques closely resembles the various manifestations of human M. tuberculosis infection. Infect. Immun. 71, 5831-44 (2003).

3. Maiello, P. et al. Rhesus macaques are more susceptible to progressive tuberculosis than cynomolgus macaques: A quantitative comparison. Infect. Immun. 86, (2018).

4. Sharpe, S. et al. Ultra low dose aerosol challenge with Mycobacterium tuberculosis leads to divergent outcomes in rhesus and cynomolgus macaques. Tuberculosis 96, 1-12 (2016).

5. Fletcher, H. a et al. Human newborn bacille Calmette-Guérin vaccination and risk of tuberculosis disease: a case-control study. BMC Med. 14, 76 (2016).

6. Sibley, L. et al. Differences in monocyte: lymphocyte ratio and Tuberculosis disease progression in genetically distinct populations of macaques. Sci.

Rep. 9, 3340 (2019). 
7. Muller, J., Tanner, R., Matsumiya, M. \& Snowden, M. A. Cytomegalovirus infection is a risk factor for TB disease in Infants. 1-49 (2017).

8. Webster, R. L. \& Johnson, R. P. Delineation of multiple subpopulations of natural killer cells in rhesus macaques. Immunology 115, 206-214 (2005).

9. Sharpe, S. A. et al. Determination of lesion volume by MRI and stereology in a macaque model of tuberculosis. Tuberculosis 89 , 405-416 (2009).

10. Elhmouzi-Younes, J. et al. In depth comparative phenotyping of blood innate myeloid leukocytes from healthy humans and macaques using mass cytometry. Cytom. Part A 91, 969-982 (2017).

11. Bjornson-Hooper, Z. B. et al. A comprehensive atlas of immunological differences between humans, mice and non-human primates. bioRxiv 574160 (2019). doi:10.1101/574160

12. WHO. Tuberculosis: Co-epidemics of TB and HIV - Data by WHO region. Global Health Observatory (2014). Available at: http://apps.who.int/gho/data/view.main.TBHIVWHOREG?lang=en. (Accessed: 9th July 2014)

13. Chen, C. Y. et al. A critical role for CD8 T cells in a nonhuman primate model of tuberculosis. PLoS Pathog. 5, e1000392 (2009).

14. Zitsman, J. S. et al. Distinctive leukocyte subpopulations according to organ type in cynomolgus macaques. Comp. Med. 66, 308-323 (2016).

15. Wolday Id, D. et al. Role of CD4/CD8 ratio on the incidence of tuberculosis in HIV-infected patients on antiretroviral therapy followed up for more than a decade. (2020). doi:10.1371/journal.pone.0233049

16. Harris, L. D. et al. Distinct Human NK Cell Phenotypes and Functional Responses to Mycobacterium tuberculosis in Adults From TB Endemic and Nonendemic Regions. Front. Cell. Infect. Microbiol. 10, 120 (2020).

17. Dijkman, K. et al. Disparate Tuberculosis Disease Development in Macaque Species Is Associated With Innate Immunity Infection Take After Repeated Exposure to. 10,1-18 (2019).

18. Roberts, L. L. \& Robinson, C. M. Mycobacterium tuberculosis infection of human dendritic cells decreases integrin expression, adhesion and migration to chemokines. Immunology 141, 39-51 (2014).

19. Uehira, K. et al. Dendritic cells are decreased in blood and accumulated in granuloma in tuberculosis. Clin. Immunol. 105, 296-303 (2002).

20. Blomgran, R., Desvignes, L., Briken, V. \& Ernst, J. D. Mycobacterium tuberculosis inhibits neutrophil apoptosis, leading to delayed activation of naive CD4 T cells. Cell Host Microbe 11, 81-90 (2012).

21. Kanthaswamy, S. et al. The genetic composition of populations of cynomolgus macaques (Macaca fascicularis) used in biomedical research. J. Med. Primatol. 42, 120-131 (2013).

22. Mee, E. T. et al. MHC haplotype frequencies in a UK breeding colony of Mauritian cynomolgus macaques mirror those found in a distinct population from the same geographic origin. J. Med. Primatol. 38, 1-14 (2009).

23. Mitchell, J. L., Mee, E. T., Almond, N. M., Cutler, K. \& Rose, N. J. Characterisation of MHC haplotypes in a breeding colony of Indonesian cynomolgus macaques reveals a high level of diversity. Immunogenetics 64, 123-129 (2012).

24. Kleiveland, C. \& Kleiveland, C. Peripheral blood mononuclear cells. in The Impact of Food Bioactives on Health: In Vitro and Ex Vivo Models $161-167$ (Springer International Publishing, 2015). doi:10.1007/978-3-319-16104-4_15

\section{Figures}


A
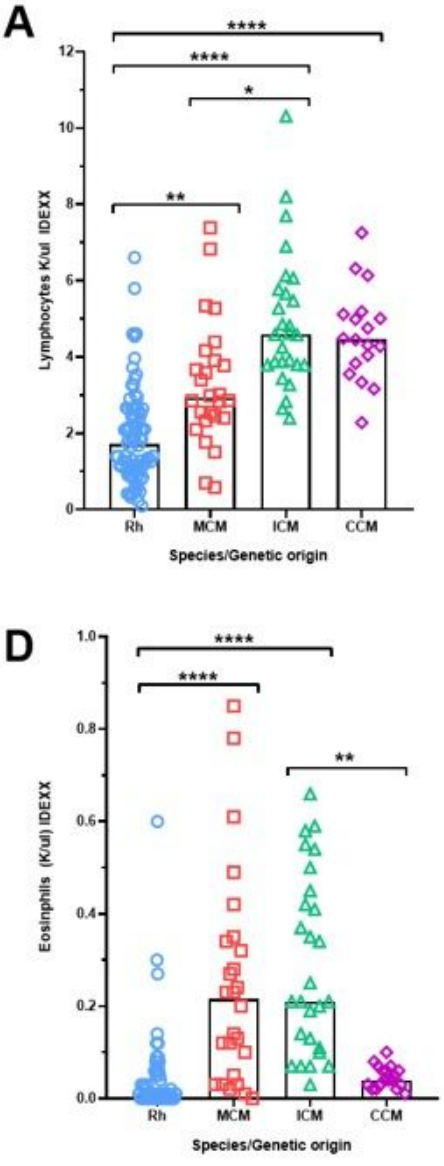

B

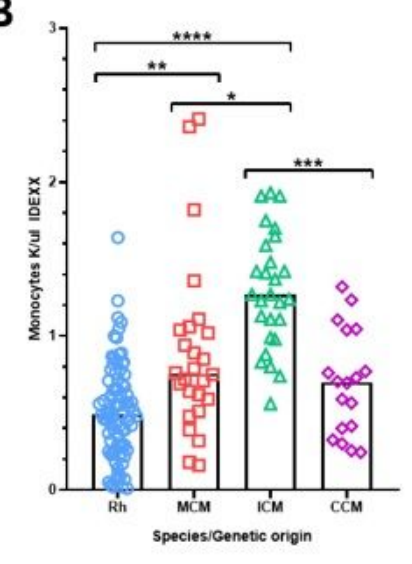

E

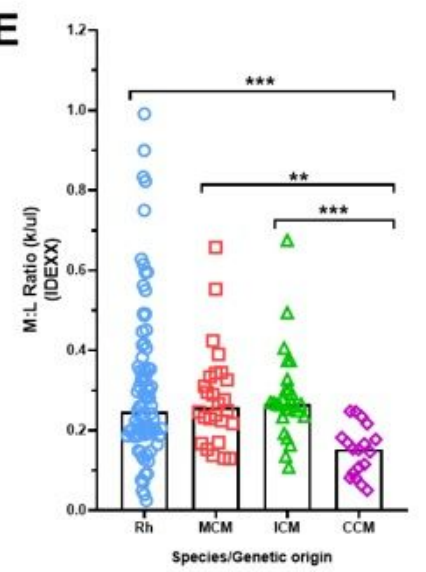

C
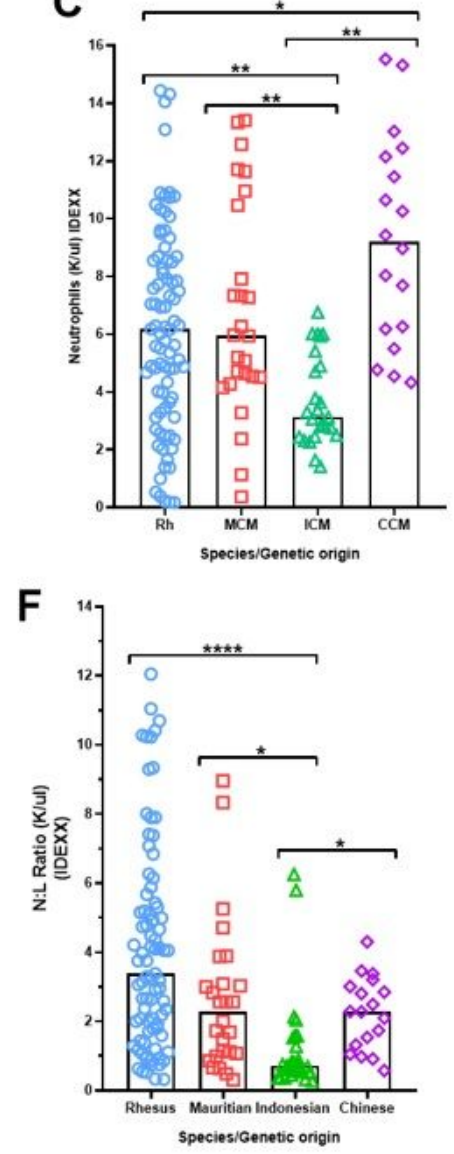

Figure 1

Comparison of immune cell populations and ratios between macaques of distinct genetic origin, as measured using IDEXX ProCyte Haematology analyser. A) Lymphocytes, B) Monocytes, C) Neutrophils, D) Eosinophils, E) M:L, F) N:L. Cell populations expressed in absolute counts K/ $\mu \mathrm{L}$. Ratios calculated from cell populations expressed in absolute counts $(K / \mu l)$. Each data point represents an individual animal: Rhesus = Blue $(n=84)$, Mauritian = Red $(n=26)$, Indonesian $=$ Green $(n=27)$, Chinese $=$ Purple $(n=18)$, bars show the group median. Kruskall-Wallis tests with Dunn's multiple comparisons were applied, as not all groups passed Shapiro-Wilk test for normality $\left(\mathrm{p}=0.05\right.$. ${ }^{*} \mathrm{p}<$ (or equal) $0.05,{ }^{\star *} \mathrm{p}<0.01$ and ${ }^{\star * \star} \mathrm{p}<0.001$. 

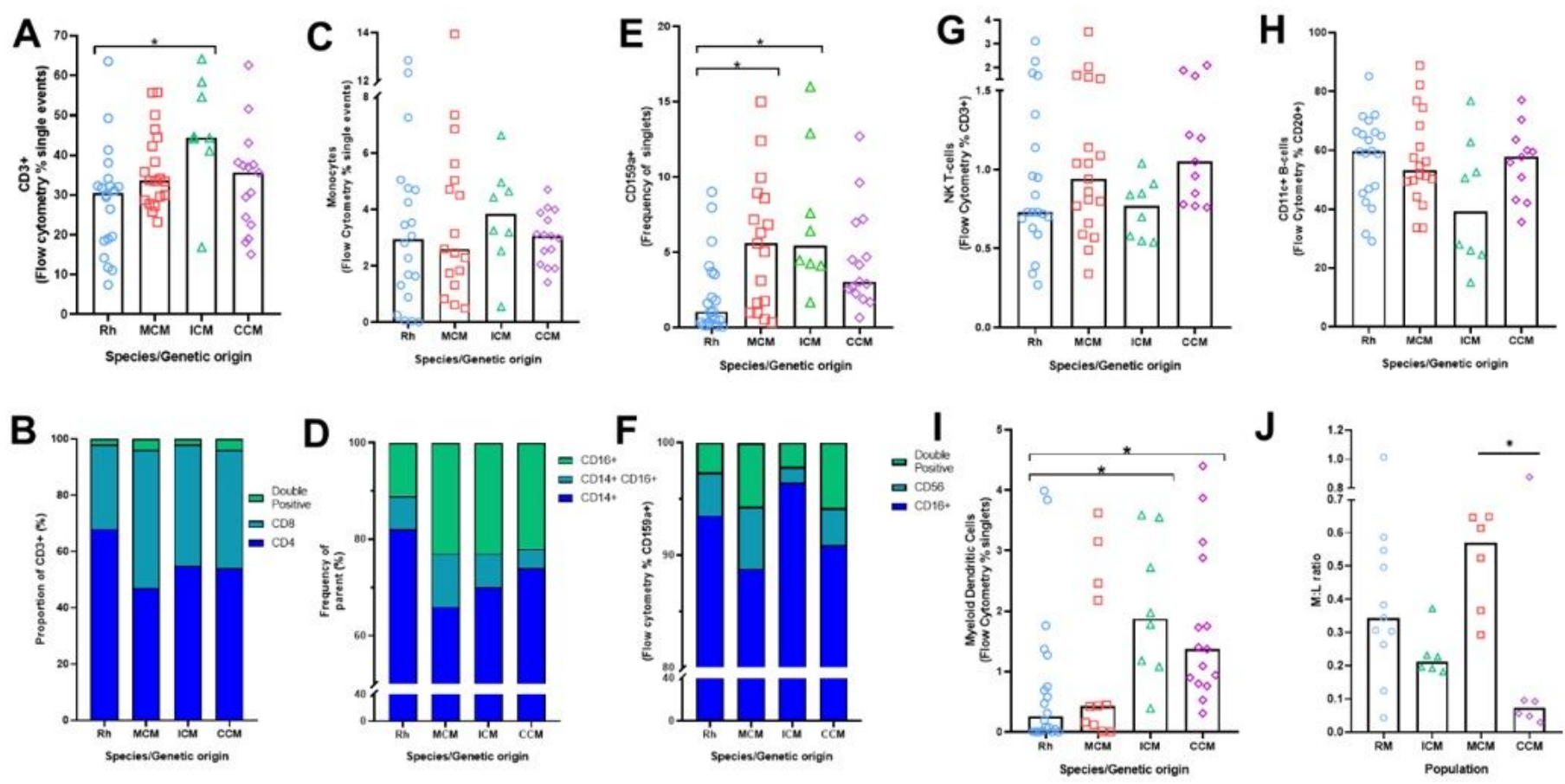

Figure 2

Cell populations in PBMCs determined by flow cytometric analysis in rhesus and cynomolgus macaques. A) CD3+ lymphocytes, B) proportions of CD4+, CD8+ and CD4+CD8+ lymphocytes, C) Monocytes, D) proportions of classical (CD14+ CD16-), intermediate (CD14+ CD16+) and non-classical (CD14- CD16+) monocytes, E) CD159+ NK cells, F) proportions of cytotoxic (CD16+ CD56-), intermediate (CD16+ CD56+) and immunomodulatory (CD16- CD56+) NK cells, G) NK T-cells, H) CD11 c+ B-cells, I) mDCs, J) M:L ratio. Each data point represents an individual animal. Bars represent median values in graphs B-F. Group numbers varied between comparisons, as previous immunophenotyping data for Indonesian and Chinese cynomolgus macaques was used to bolster comparisons: Rhesus (Blue) $n=20$ and MCM (Red) n=19. For comparisons A, B and D, ICM (Green) n=18 and CCM (Purple) $n=22$. For comparisons C, E and F, ICM (Green) $n=8$ and CCM (Purple) $n=15$. Kruskall-Wallis tests with Dunn's multiple comparisons were applied, ${ }^{*} p=0.05,{ }^{* \star} p<0.01$ and ${ }^{\star * \star} p<0.001$.
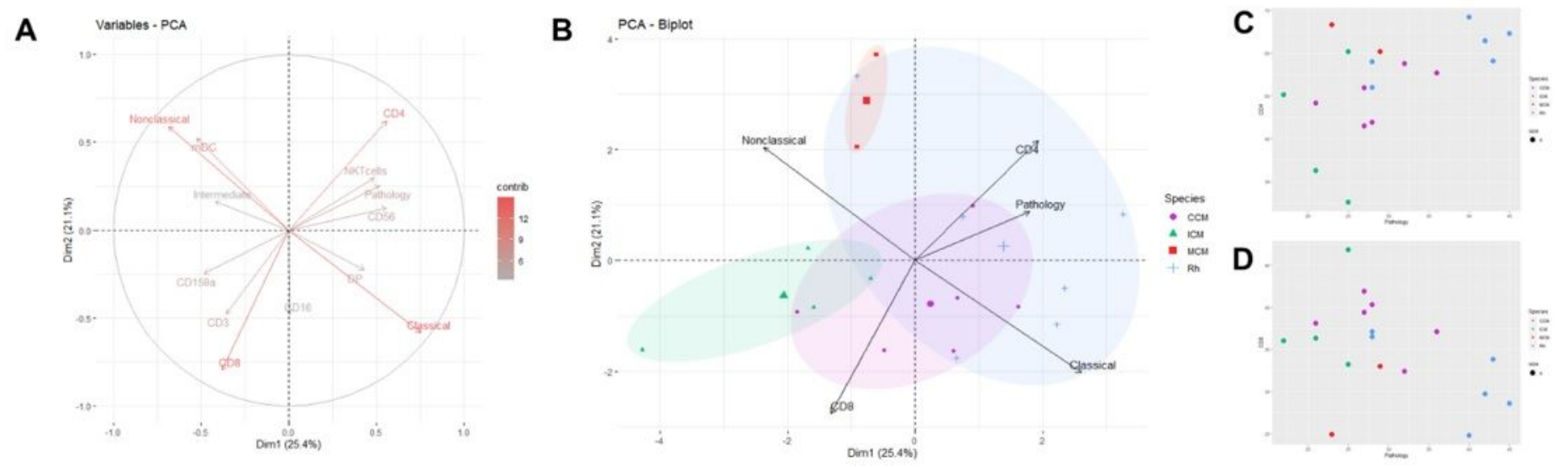

\section{Figure 3}

Principle component analysis of immune parameters between macaque groups using the immunophenotyping data and correlations with pathology scores. A) PCA loading plot of immune parameters, with a scale for relative contribution of each component to the model. Red = highest to grey = lowest, B) PCA biplot showing how the macaque populations cluster towards the variables that contribute the most to the variation in the data. Largest of each shape indicates the mean of each population with smaller shapes indicating the outliers. Blue circles = CCM, yellow triangles = ICM, grey squares = MCM, red crosses = RM C) Correlation of CD4+ T-cells with pathology scores following TB infection, with each population indicated by a different colour, red = CCM, green $=I C M$, blue $=$ MCM and red $=$ RM D) Correlation of CD8+ T-cells with pathology scores following TB infection, with each population indicated by a different colour, red $=\mathrm{CCM}$, green $=\mathrm{ICM}$, blue $=\mathrm{MCM}$ and red $=\mathrm{RM}$. 


\section{Supplementary Files}

This is a list of supplementary files associated with this preprint. Click to download.

- Supplementarydata.docx 